\title{
Androstenedione changes steroidogenic activity of SGBS cells
}

\author{
Jana Ernst ${ }^{1}$, Katharina Gert ${ }^{1}$, Frank Bernhard Kraus ${ }^{2}$, Ulrike Elisabeth Rolle-Kampczyk ${ }^{3}$, Martin Wabitsch ${ }^{4}$, \\ Faramarz Dehghani ${ }^{1}$ and Kristina Schaedlich ${ }^{1}$ \\ 1'Department of Anatomy and Cell Biology, Faculty of Medicine, Martin Luther University Halle-Wittenberg, Grosse Steinstrasse, Halle (Saale), Germany \\ ${ }^{2}$ Central Laboratory, University Hospital Halle (Saale), Ernst-Grube-Strasse, Halle (Saale), Germany \\ ${ }^{3}$ Department of Molecular Systems Biology, Helmholtz Centre for Environmental Research Leipzig, Leipzig, Germany \\ ${ }^{4}$ Division of Pediatric Endocrinology and Diabetes, Department of Pediatrics and Adolescent Medicine, University Medical Center Ulm, Ulm, Germany
}

Correspondence should be addressed to J Ernst: jana.ernst@medizin.uni-halle.de

\begin{abstract}
The rapid increase of obesity during the last decades and its future prospects are alarming. Besides the general discussed causes of obesity, the 'Developmental Origins of Health and Disease' (DOHaD) hypothesis received more attention in recent years. This hypothesis postulates an adverse influence during early development that programs the unborn child for metabolic dysfunctions later in life. Childhood obesity - an as much increasing problem - can be predisposed by maternal overweight and diabetes. Both, obesity and hyperinsulinemia are major causes of female hyperandrogenemia. As predicted by the DOHaD hypothesis and shown in animal models, developmental androgen excess can lead to metabolic abnormalities in offspring. In this study, we investigated, if androgen exposure adversely affects the adipogenic differentiation of preadipocytes and the endocrine function of adult adipocytes. The human SGBS preadipocyte model was used to affirm the de novo biosynthesis of steroid hormones under normal adipogenesis conditions. Normal adipogenesis was paralleled by an increase of corticosteroids and androgens, whereas estrogen remained at a steady level. Treatment with androstenedione had no effect on SGBS proliferation and differentiation, but adult adipocytes exhibited a significant higher accumulation of triglycerides. Progesterone (up to 2-fold), testosterone (up to 38-fold) and cortisone (up to 1.4-fold) - but not cortisol - were elevated by androstenedione administration in adult adipocytes. Estrogen was not altered. Data suggest that androgen does not negatively influence adipogenic differentiation, but steroidogenic function of SGBS adipocytes.
\end{abstract}

\section{Introduction}

Across the OECD area more than one in five adults and nearly one in six children are overweight (BMI $\geq 25 \mathrm{~kg} / \mathrm{m}^{2}$ ) or obese (BMI $\geq 30 \mathrm{~kg} / \mathrm{m}^{2}$ ), and a steady increase in obesity rates until 2030 is expected (1). Obesity and its comorbidities are symptoms of the metabolic syndrome, that currently is one of the main social and public health problems. In fact, the worldwide epidemic of overweight and obesity, its rapid increase over the last decades and its future prospects has been termed as 'globesity' by the
World Health Organization (WHO) (2). In causal research the 'Developmental Origins of Health and Disease' (DOHaD) hypothesis has attracted more and more attention during the last years. It postulates that an adverse influence during early development (i.e. preconceptional up to 1000 days of postnatal life) can program the unborn/newborn for metabolic dysfunctions later in life. Apart from environmental factors maternal diseases such as overweight or excessive weight gain during pregnancy

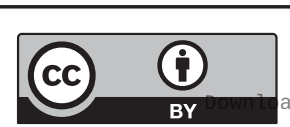

This work is licensed under a Creative Commons Attribution 4.0 International License. 
and diabetes (type 1 , type 2 or gestational) are among the adverse influences put forward by this hypothesis $(3,4,5)$.

Obesity and hyperinsulinemia are major causes for female hyperandrogenemia. The thereby elevated androgen levels in the circulation result from an exceeding and dysregulated production of androgens by classical steroidogenic organs $(6,7)$. Classical steroidogenic organs such as the adrenal gland and the gonads are characterized by the presence of an enzyme machinery enabling them for the de novo synthesis of steroid hormones from cholesterol (8). Androgenic steroids exhibit a positive gradient from blood to adipose tissue with the precursor androstenedione and dehydroepiandrosterone as well as the active androgen testosterone, being the most abundant in the circulation (9). However, it has also been hypothesized that a further and substantial contributor to androgen production is adipose tissue (10). Besides the body's own endocrine dysregulations, an environmental exposure to androgens exists due to the prevalent contamination of surface water and groundwater (11, $12,13)$. Androgen excess during early development is hypothesized to program metabolic abnormalities in male and female offspring as demonstrated in mice $(14,15,16)$ and rhesus monkeys $(17,18,19)$ as wells as in humans $(17,18,19,20,21)$.

Changes in the levels of circulating steroid hormones influence the steroidogenic activity of the adipose tissue and may result in an adipose tissue dysfunction with abnormal adipokine synthesis and disturbance of the lipid homeostasis and adipogenesis (22). Synthesis and metabolism of steroid hormones are regulated by a complex network of steroidogenic enzymes. Studies on this topic so far showed partly contradictory results due to investigations on isolated aspects of the network, differences between species and sexes as well as specificities of fat depots $(21,22,23,24)$.

Only a few studies have dealt with the impact of androgens on steroid hormone synthesis in human adipose tissue so far. Wabitsch and colleagues could show that human Simpson-Golabi-Behmel syndrome (SGBS) preadipocytes are comparable to in vitro matured adipocytes from human s.c. fat and as such are a good model system $(25,26)$. By demonstrating the conversion of cholesterol to pregnenolone as the initial step of steroid biosynthesis in the SGBS model, the evidence for adipocytes as steroidogenic cells was established as well (27). Additionally, the ability to secrete adipokines like leptin and adiponectin $(28,29,30)$ provides it for the investigation of the endocrine function of (pre)adipocytes during adipogenesis and for the characterization of interactions of endocrine signaling. In the present study, we studied the steroidogenic activity of SGBS adipocytes followed by the investigation of the impact of androstenedione as a precursor of active steroid hormones on adipogenesis, steroid hormone synthesis and the adipokine system.

\section{Material and methods}

\section{Cell culture}

SGBS preadipocytes, a non-immortalized cell model for adipogenic differentiation, were cultured and differentiated to mature adipocytes as described earlier $(25,26)$. Culture medium was changed at day 4 of adipogenesis replacing the induction medium by the differentiation medium. Application of $10 \mu \mathrm{M}$ 4-androstene-3,17-dione (SigmaAldrich) occurred from day 0 to day 8 of adipogenesis. Samples for mRNA expression and hormone measurement were taken at day $0,2,4,6$ and 8 .

\section{Western blot analyses}

To investigate the possible effects of androstenedione on cell proliferation, SGBS preadipocytes were cultured in basal medium for 2 days before adipogenic induction (26). Thereafter, androstenedione was applied for $24 \mathrm{~h}$. For Western blot analyses, the SGBS cells were harvested and lysed in RIPA buffer containing 10x PBS, Nonidet NP40, 10\% SDS, 0.5\% sodiumdeoxycholate, protease and phosphatase inhibitors (Roche). Separation of total protein lysates by SDS-PAGE and the transfer to nitrocellulose membranes were performed. Blots were blocked in $0.1 \%$ TBST with 3\% (wt/vol) BSA for $2 \mathrm{~h}$. The primary antibodies against proliferating-cell-nuclear-antigen (PCNA) (1:1000; Cell Signaling) and $\beta$-ACTIN (1:10,000; Sigma Aldrich) were incubated at $4^{\circ} \mathrm{C}$ overnight. Afterwards, the secondary horseradish peroxidase-conjugated antibody goat anti-mouse (1:20,000; Dianova, Hamburg, Germany) was applied for $1 \mathrm{~h}$ at room temperature.

For the immunodetection of proteins at $\mathrm{d} 8$ of adipogenesis the procedure of cell lysis, protein separation and blot preparation was performed as described above. The primary antibodies against HSL (1:500; Cell Signaling), pHSL (1:250; Cell Signaling) and Perilipin (1:500; Cell Signaling) were incubated at $4^{\circ} \mathrm{C}$ overnight. Afterwards the secondary horseradish peroxidase (HRP)-conjugated antibodies goat anti-mouse (1:10,000; Dianova) or goat anti-rabbit (1:6000; Dako)

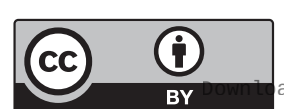

This work is licensed under a Creative Commons Attribution 4.0 International License. 
were applied for $1 \mathrm{~h}$ at room temperature. The primary antibody against GAPDH (1:1000; Cell Signaling) was already conjugated to horseradish peroxidase and did not require a secondary antibody. Detection was performed with the immobilon western chemiluminescent HRP substrate (MerckMillipore). Subsequently, for analyses the ChemiDoc $^{\text {TM }}$ Touch MP Imaging System (BioRad) and the Image Lab 6.0 software were used. The protein amount was calculated as the ratio of each protein vs $\beta$-ACTIN or GAPDH intensity.

\section{Quantitative real-time PCR}

Quantitative real-time PCR (qRT-PCR) was carried out on a StepOnePlus ${ }^{\mathrm{TM}}$ real-time PCR system (Applied Biosystems) to measure mRNA expression levels in the SGBS preadipocytes. Transcription with normalisation to $10^{3}$ copies of the TATA-box-binding protein (TBP) as housekeeping gene was determined for the following genes: AR, CYP11A1, CYP17A1, CYP19, ER $\alpha, E R \beta$, GLUT4, GPER, GR, HSD3B, HSD11B1, HSD17B5, PGR and StAR. The specifications of primers and amplicons are given in Table 1. Quantitative standard curves were generated for each gene using a plasmid dilution series containing the target sequences $\left(10^{3}-10^{6}\right.$ copies).

\section{Hormone assays}

Cell supernatants were collected and concentrations of the adipokines leptin (high-sensitive leptin ELISA, IBL, Hamburg, Germany) and adiponectin (Quantikine ${ }^{\circledR}$ ELISA Human Total Adiponectin/Acrp30, BioVendor,
Kassel, Germany) were measured by ELISA according to manufacturer's manual. ELISA data was normalized to the total protein concentration of individual sample. Protein was isolated using radioimmunoprecipitation assay (RIPA) buffer and the concentration was determined by the BioRad Protein Assay (BioRad). Furthermore, an ELISA kit (DRG Instruments, Marburg, Germany) was used for determining the concentration of cortisone.

Cholesterol and steroid hormones (cortisol, DHEAS, estradiol, progesterone, testosterone) were analyzed in the Central Laboratory of the University Hospital Halle. Cholesterol was measured using a colorimetric assay (CHOL2 Cholesterol Gen.2, Roche Diagnostics) on a Roche cobas c701 Analyzer integrated in a fully automated Roche Cobas 8000 platform. Cortisol, DHEAS, estradiol, progesterone and testosterone were measured with electrochemiluminescence Immunoassays (Elecsys Cortisol II, DHEA-S, Elecsys Progesterone III, Elecsys Testosterone II and Elecsys Estradiol III, Roche Diagnostics) on a Roche Cobas e602 Analyzer, also being part of the fully automated Roche Cobas 8000 platform. All analyses on the Roche Cobas Analyzers were carried out according to the manufacturer's instructions and manuals, with routine maintenance and quality control procedures.

\section{LC-MS/MS}

Steroids in SGBS cell supernatants were measured in the Department of Molecular Systems Biology of the Helmholtz-Centre for Environmental Research - UFZ Leipzig using the AbsoluteIDQ ${ }^{\circledR}$ Stero17 Kit

Table 1 Primers for quantitative RT-PCR.

\begin{tabular}{|c|c|}
\hline Gene & Accession number \\
\hline$A R$ & NM_000044 \\
\hline CYP19 & NM_000103 \\
\hline CYP11A1 & NM_000781 \\
\hline$E R a$ & NM_000125 \\
\hline$E R \beta$ & NM_001437 \\
\hline GLUT4 & NM_001042 \\
\hline GPER & NM_001505 \\
\hline$G R$ & NM_000176 \\
\hline$H S D 3 B$ & NM_000198 \\
\hline HSD11B1 & NM_005525 \\
\hline HSD17B5 & NM_003739 \\
\hline PGR & NM_001202474 \\
\hline StAR & NM_000349 \\
\hline$T B P$ & NM_003194 \\
\hline
\end{tabular}

\begin{tabular}{l} 
Forward primer \\
\hline AATTCCTGTGCATGAAAGCCA \\
ATGTGGACGTGTTGACCCTTCT \\
CTCAGTCCTGGTCAAAGGCT \\
CAATGACTATGCTTCAGGCTAC \\
AGCCACCATGAATATCCAGCCA \\
ACTGGCCATTGTTATCGGCA \\
ATGACCATCCCCGACCTGTA \\
ACTGCTTCTCTCTTCAGTTC \\
GGAAGCCAAGCAGAAAACCG \\
CTCTACAGAAGGTGGTATCC \\
TGAGGAGAAGCAGCAGCAAA \\
ATTCACTTTTTCACCAGGTC \\
GGCCTTGGGCATCCTTAG \\
TGTGCTCACCCACCAACAAT
\end{tabular}

\begin{tabular}{l} 
Reverse primer \\
\hline AGCTCTCTCGCAATAGGCTG \\
AGGAGAGCTTGCCATGCATCAA \\
CTTCTCCCTGTAAATCGGGC \\
CCACCTTTCATCATTCCCAC \\
TGGCCACAACACATTTGGGCTT \\
GTCAGGCGCTTCAGACTCTT \\
GAGGAAGAAGACGCTGCTGT \\
GATTTTCAACCACTTCATGC \\
GCCCCTGTTGCCTTCTGTAT \\
AATGAGCATGTCTAGTCCTC \\
GGCGGAACCCAGCTTCTATT \\
AACCTGGCAATGATTTAGAC \\
TCCACCACGACCTCCAG \\
AGTCGTCTTCCTGAATCCCT
\end{tabular}

\begin{tabular}{cccc}
\hline $\mathbf{T m}\left({ }^{\circ} \mathrm{C}\right)$ & & Amplicon $(\mathrm{bp})$ \\
\cline { 1 - 1 } 58 & & 211 \\
60 & & 133 \\
63 & & 250 \\
60 & & 198 \\
60 & & 129 \\
60 & & 213 \\
60 & & 174 \\
60 & & 168 \\
58 & & 172 \\
61 & & 142 \\
60 & & 190 \\
60 & & 180 \\
60 & & 131 \\
60 & & 199 \\
\hline
\end{tabular}

$A R$, androgen receptor; CYP11A1, cholesterol side-chain cleavage enzyme P450scC; CYP19, cytochrome P450 aromatase; ERa and ER $\beta$, estrogen receptor alpha and beta; GLUT4, glucose transporter 4; GPER, G protein-coupled estrogen receptor 1; GR, glucocorticoid receptor; HSD11B1, 11beta-hydroxysteroid dehydrogenase 1; HSD17b5, 17beta-hydroxy steroid dehydrogenase 5; HSD3B, 3beta-hydroxysteroid dehydrogenase; PGR, progesterone receptor; TBP, TATA-box-binding protein.

https://ec.bioscientifica.com https://doi.org/10.1530/EC-19-0549 (c) 2020 The authors Published by Bioscientifica Ltd

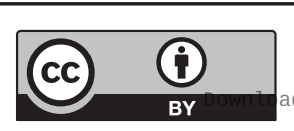

This work is licensed under a Creative Commons Attribution 4.0 International License. ded from Bioscientifica.com at 04/26/2023 01:00:50PM 
(Biocrates Life Sciences AG, Innsbruck, Austria). Sample preparation was performed by manufacturer's instructions executed by a solid phase extraction procedure. The LC-MS/MS analyses were carried out by MRM acquisition (electrospray ionization, positive ionization mode) using a Waters Acquity UPLC System (Waters GmbH, Eschborn, Germany) coupled with Q-TRAP 5500 (Sciex, Darmstadt Germany GmbH). The quantification of LC-MS/MS generated data was performed by IntelliQuan algorithm from Analyst 1.6.2 software (Sciex) and evaluated by MetIDQ software (Biocrates).

\section{Immunohistochemistry for the localization of GLUT4}

For the in situ immunodetection of GLUT4, SGBS adipocytes were cultured on chamber slides (Sarstedt, Nümbrecht, Germany). At day 8 cells were fixed with $4 \%$ paraformaldehyde for $25 \mathrm{~min}$. After a washing step with PBS, endogenous peroxidases were blocked with $\mathrm{H}_{2} \mathrm{O}_{2}$ (3\% in methanol) for $20 \mathrm{~min}$. Further washing steps with tap water and PBST followed before the blocking with goat serum (10\% in PBST) for $1 \mathrm{~h}$. The primary antibody against GLUT4 (1:300; Sigma Aldrich) was incubated at $4^{\circ} \mathrm{C}$ overnight. Afterwards the secondary HRP-conjugated antibody goat anti-rabbit (1:1; Dako) was applied for $90 \mathrm{~min}$ at room temperature. Detection was performed with 3,3'-diaminobenzidin and hematoxylin was used for nuclear counterstaining.

\section{Triglyceride assay}

The 'Adipogenesis Kit' (Sigma Aldrich) was used to detect the total cellular concentrations of triglycerides according to manufacturer's manual. The coupled enzyme assay results in a colorimetric product, proportional to the trigylcerides present at day 8 of adipogenesis. Analyses were performed with the Synergy ${ }^{\mathrm{TM}} \mathrm{Mx}$ microplate reader (BioTeK Inc., Winooski, VT, USA). Data were normalized to the protein concentration of each individual sample. Protein was isolated using radioimmunoprecipitation assay (RIPA) buffer and concentrations were determined by the BioRad Protein Assay (BioRad).

\section{Statistical analyses}

At least four independent experiments $(\mathrm{N})$ were performed with 12 wells pooled per treatment and experiment. Data were presented as mean \pm S.E.M. Results of adipogenesis experiments were investigated by the ANOVA with the
Bonferroni's post hoc test in relation to the control group. To evaluate differences between the treatment with and without androstenedione (AD) an unpaired Student's $t$-test or if necessary the Wilcoxon rank-sum test was used. Data differences were considered as statistically significant at $P$ value $\leq 0.05$.

\section{Results}

\section{Androstenedione does not affect SGBS cell proliferation and differentiation}

Cell proliferation of SGBS preadipocytes was investigated by determining the PCNA amount, but no influence of androstenedione was detectable (Fig. 1A). Furthermore, adipogenic differentiation was evaluated by measuring the secretion of the adipokines leptin and adiponectin in cell supernatants during adipogenesis (Fig. 1B). As expected, both adipokines increased during normal adipogenesis compared to day 0. Leptin was not detectable at day 0 and day 2, but significantly elevated at days 4,6 and 8 . The slight decline from day 4 to day 6 is not significant. With day 8 the highest level of leptin was measured reaching significant value also compared to day 4 and day 6. Adiponectin showed significantly elevated values at day 8 (up to the 25 -fold compared to day 0 ) only. After the application of androstenedione the levels of both adipokines were not significantly different compared to the corresponding control samples of the same day (Fig. 1B). The investigation of several adipogenic and differentiation related genes such as PPARs, GLUT4, FABP4 and CD36 revealed no effect on SGBS adipogenesis by androstenedione. Furthermore, we investigated the localization of GLUT4 within adult adipocytes (d8). Its inactive form is accumulated in the membrane of the Golgi apparatus. Due to an insulin-dependent activation the glucose transporter is translocated to the cell membrane. A positive staining was detectable in the cytoplasm of the adipocytes. However, the more intensely stained adipocyte cell lining recommends a predominant membrane-bound distribution that was not changed by androstenedione (Fig. 1C).

\section{Androstenedione causes accumulation of triglycerides}

The triglyceride content within the adult adipocytes (day 8) was measured revealing 1.4-fold higher triglyceride levels in the cells treated with androstenedione (Fig. 2A). Additionally, we analyzed the amount of PLIN1 and HSL as

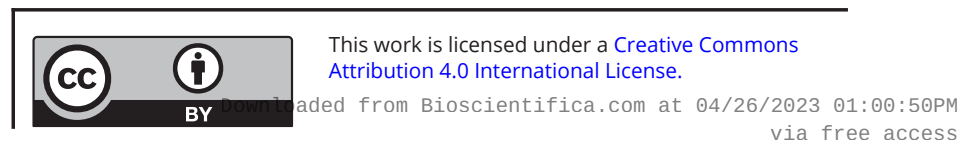



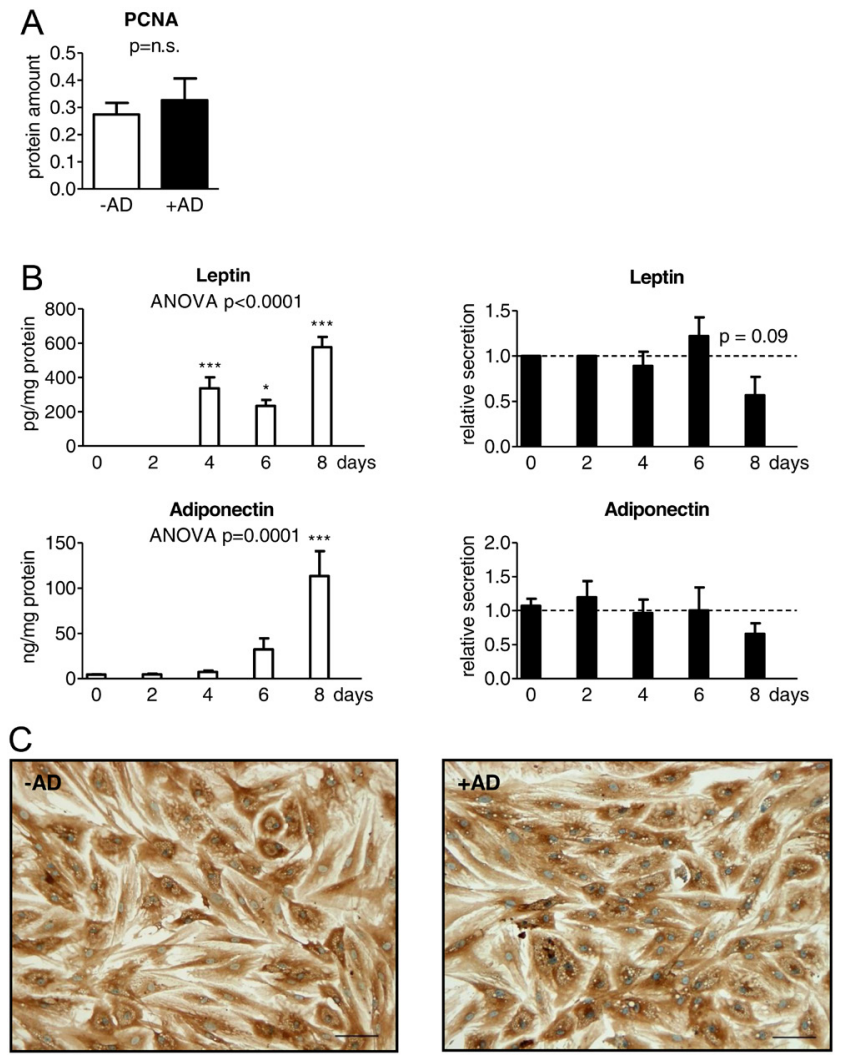

\section{Figure 1}

Influence of androstenedione on SGBS cell proliferation and differentiation. (A) SGBS cell proliferation was analyzed in SGBS preadipocytes before induction of adipogenic differentiation by determination of the amount of the proliferating-cell-nuclear-antigen (PCNA) under normal conditions and with application of androstenedione (AD) normalized to $\beta$-Actin ( $n=4 ; P \leq 0.05^{\#}$ for comparing unexposed and AD-exposed cells). (B) For SGBS cell differentiation, secretion of leptin and adiponectin was measured during adipogenic differentiation under normal conditions (left). Additionally, AD stimulation is described in relation to the corresponding $A D$-free control level indicated as dotted line (right) $(n=4 ; * P \leq 0.05 ; * \star P \leq 0.01 ; * \star * P \leq 0.001$ for normal adipogenesis in comparison to day 0 and $\# \leq \leq 0.05$; \# $P \leq 0.01$; $\# \#$ \# 0.001 for comparing unexposed and AD-exposed cells). (C) For investigating the localization of GLUT4, immunodetection (brown staining) of this glucose transporter was performed in-situ of adult adipocytes (day 8) with nuclear counterstaining by hematoxylin (blue staining) comparing unexposed and AD exposed. The scale bar indicates $100 \mu \mathrm{M}$.

well as the activation of HSL by determining the quotient of the phosphorylated to the non-phosphorylated protein, but no alteration by androstenedione was detectable for both the protein amount and the activation (Fig. 2B, C and D).

\section{Androstenedione stimulates the secretion of progesterone, testosterone and cortisone}

Cholesterol and steroid hormone levels were determined during adipogenesis in supernatants of SGBS cells under
A triglyceride content

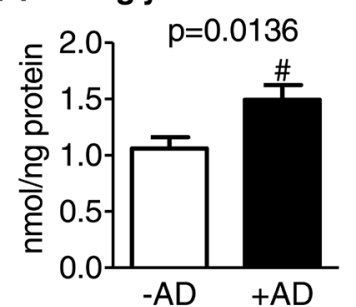

B

PLIN1

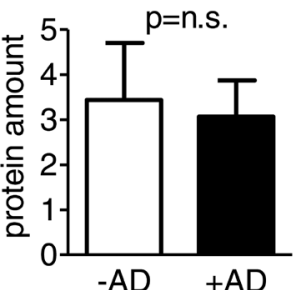

C

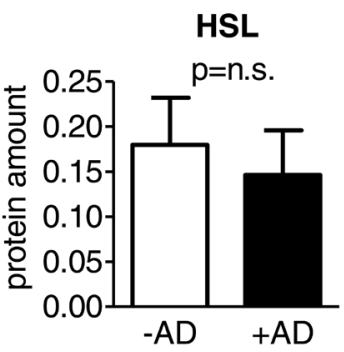

\section{Figure 2}

Influence of androstenedione on the lipid content within adult SGBS adipocytes. (A) The triglyceride content within adult SGBS adipocytes of day 8 was measured $\left(n=16 ; P \leq 0.05^{\#}\right.$ for comparing \pm AD). Furthermore, the protein amount of (B) perilipin (PLIN1) normalized to $\beta$-ACTIN and (C) HSL as well as (D) the activation of HSL by the quotient of the phosphorylated to the non-phosphorylated protein normalized to GAPDH were determined $(n=4)$.

normal conditions and after androstenedione-treatment with immunoassays (Fig. 3) and LC-MS/MS (Table 2). During normal adipogenesis cholesterol significantly decreased in the supernatant of SGBS cells at day 4 and day 8 (by $70 \%$ at day 4 and by $57 \%$ at day 8 each compared to day 0) (Fig. 3A). Simultaneously, a significantly increased secretion of progesterone (up to 1.3-fold), cortisol (up to 7 -fold) and cortisone (up to 2 -fold) was detectable at day 4 and day 8 compared to day 0 (Fig. 3B, C and D). In addition, DHEAS showed an up to 1.1-fold increase at day 4 compared to day 0 (Fig. 3E). Estradiol and testosterone did not change during adipogenesis (Fig. 3F and 3G). Application of androstenedione caused a significant increase of progesterone up to the 1.7 -fold at day 4 and up to 2 -fold at day 8 compared to the corresponding control sample of the same day (Fig. 3B). The same was observed for testosterone with a 13-fold increase at day 4 and up to 38 -fold increase at day 8 compared to the corresponding control sample of the same day (Fig. 3G). For cortisone up to 1.4-fold increase compared to the control sample of the same day could only be observed at day 8 (Fig. 3D).

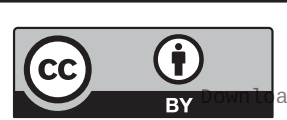



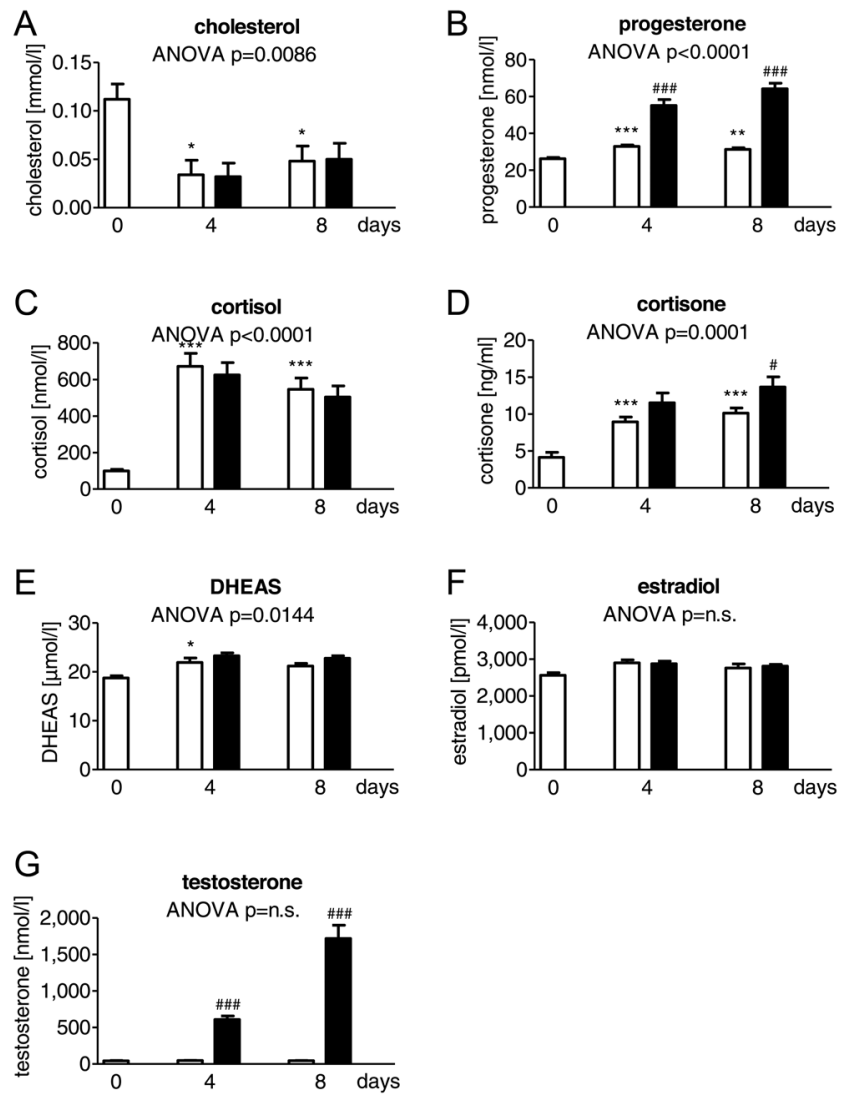

\section{Figure 3}

Influence of androstenedione on the steroid hormone synthesis in the SGBS cell model during adipogenesis. (A) Cholesterol, (B) progesterone, (C) cortisol, (D) cortisone, (E) dehydroepiandrosterone sulfate (DHEAS), (F) estradiol and (G) testosterone were determined in SGBS cells during adipogenic differentiation in controls (white columns) and with $A D$ stimulation (black columns) $(n=5 ; * P \leq 0.05 ; * * P \leq 0.01 ; * \star * P \leq 0.001$ for normal adipogenesis in comparison to day 0 and ${ }^{\#} P \leq 0.05$; \#\# $P \leq$ 0.01 ; \#\# $P \leq 0.001$ for comparing unexposed and AD-exposed cells).

The above-mentioned findings were replicated by LC-MS/MSand additionalsteroidhormoneswere analyzed. Under normal conditions, androgens (androstenedione, androsterone, testosterone, dihydrotestosterone, etiocholanolone) and corticosteroids (corticosterone, aldosterone, 11-deoxycortisol, cortisol, cortisone) increased during adipogenesis. Furthermore, an androstenedione-mediated increase was found for androsterone, 11-deoxycortisol, dihydrotestosterone and etiocholanolone at day 8 (data are provided in Table 2).

\section{Androstenedione upregulates the enzyme for cortisol-cortisone interconversion}

The transcription of enzymes important for the synthesis and the metabolism of steroid hormones were investigated under normal conditions and after androstenedione treatment. SGBS cells expressed the machinery of the de novo steroid biosynthesis from cholesterol, namely StAR (Fig. 4A) and CYP11A1 (Fig. 4B). Furthermore, SGBS cells expressed HSD11B1 for the cortisol-cortisone interconversion (Fig. 4C), the aromatase CYP19 (Fig. $4 \mathrm{D})$ and the enzyme for the androgen interconversion HSD17B5 (Fig. 4E). StAR (Fig. 4A) and CYP19 (Fig. 4D) showed no alterations compared to day 0 during normal adipogenesis. However, significant changes were observed with increasing transcription for CYP11A1 at day 8 (up to the 3 -fold at day 8 compared to day 0; Fig. 4B), HSD11B1 at day 2 and day 4 (up to the 356-fold at day 2 and up to the 474-fold at day 4 each compared to day 0; Fig. 4C) and HSD17B5 at every measuring point (up to the 54 -fold at day 2 , up to the 73 -fold at day 4 , up to the 78 -fold at day 6 and up to the 99-fold at day 8 each compared to day 0; Fig. 4E). CYP17A1 and HSD3B, both metabolizing pregnenolone to downstream steroids, were detectable by sequencing, but the expression was too low for quantification by qRT.

Androstenedione treatment only significantly affected the expression of CYP11A1 at day 6 (up to the 1.6-fold compared to the corresponding control sample of the same day; Fig. 4B) and HSD11B1 at day 8 (up to the 1.9-fold compared to the corresponding control sample of the same day; Fig. 4C).

\section{Androstenedione lowers $E R \beta$ and $A R$ transcription}

Gene expression of estrogen receptors, the androgen receptor, the progesterone receptor and the glucocorticoid receptor were studied. For estrogen and androgen receptors, we found an upregulation during normal adipogenesis: significant values were reached for $E R \alpha$ at day 8 (up to the 24-fold at day 8 compared to day 0; Fig. $5 A$ ), ER $\beta$ and GPER both at day 6 and day 8 (ER $\beta$ : up to the 135 -fold at day 6 and up to the 202-fold at day 8 each compared to day 0; Fig. 5B; GPER: up to the 5-fold at day 6 and up to the 8-fold at day 8 each compared to day 0 ; Fig. 5C) as well as $A R$ at every measuring point (up to the 6-fold at day 2, up to the 5-fold at day 4, up to the 5-fold at day 6 and up to the 7-fold at day 8 each compared to day 0; Fig. 5D). Employment of androstenedione significantly lowered the transcription of $E R \beta$ at day 8 (by $41 \%$ compared to the corresponding control sample of the same day; Fig. 5B) and $A R$ at day 6 and day 8 (by $27 \%$ at day 6 and by $37 \%$ at day 8 compared to the corresponding control sample of the same day; Fig. 5D). The glucocorticoid receptor was expressed, too, without 
Table 2 Steroid hormone analysis performed by LC-MS/MS.

\begin{tabular}{|c|c|c|c|}
\hline \multirow[b]{2}{*}{ Hormone } & \multicolumn{3}{|c|}{ Normal adipogenesis ( $\mu \mathrm{mol} / \mathrm{L})$} \\
\hline & day 0 & day 4 & day 8 \\
\hline Aldosterone & $0.005 \pm 0.002$ & $0.032 \pm 0.007^{a}$ & $0.016 \pm 0.010$ \\
\hline 17alpha-hydroxyprogesterone & $0.015 \pm 0.010$ & $0.112 \pm 0.109$ & $0.014 \pm 0.012$ \\
\hline Androstenedione & $4.957 \pm 1.260$ & $13.918 \pm 2.968^{a}$ & $3.567 \pm 0.994$ \\
\hline Androsterone & $1.014 \pm 0.272$ & $3.970 \pm 1.032^{a}$ & $0.847 \pm 0.219$ \\
\hline Corticosterone & $0.005 \pm 0.003$ & $0.291 \pm 0.037^{c}$ & $0.217 \pm 0.013$ \\
\hline Cortisol & $0.301 \pm 0.155$ & $34.853 \pm 1.926^{c}$ & $28.813 \pm 1.251^{c}$ \\
\hline Cortisone & $0.080 \pm 0.019$ & $2.112 \pm 0.137^{b}$ & $3.990 \pm 0.610^{\circ}$ \\
\hline 11-Deoxycorticosterone & $0.013 \pm 0.013$ & $0.578 \pm 0.366$ & $0.091 \pm 0.068$ \\
\hline 11-Deoxycortisol & $0.002 \pm 0.001$ & $0.054 \pm 0.013^{b}$ & $0.015 \pm 0.002$ \\
\hline DHEA & $0.080 \pm 0.080$ & $0.160 \pm 0.090$ & $0.861 \pm 0.428$ \\
\hline Dihydrotestosterone & $0.058 \pm 0.013$ & $0.122 \pm 0.020^{a}$ & $0.056 \pm 0.010$ \\
\hline Estradiol & $0.030 \pm 0.004$ & $0.113 \pm 0.040$ & $0.024 \pm 0.001$ \\
\hline Estrone & $0.760 \pm 0.701$ & $0.176 \pm 0.049$ & $0.099 \pm 0.036$ \\
\hline Etiocholanolone & $0.938 \pm 0.271$ & $3.918 \pm 1.030^{a}$ & $0.832 \pm 0.219$ \\
\hline Progesterone & $0.475 \pm 0.350$ & $2.468 \pm 1.429$ & $1.092 \pm 0.450$ \\
\hline Testosterone & $1.747 \pm 0.281$ & $4.142 \pm 0.770^{a}$ & $1.260 \pm 0.270$ \\
\hline
\end{tabular}

\begin{tabular}{c}
\hline+ AD $(\mu \mathrm{mol} / \mathrm{L})$ day 8 \\
\hline $0.026 \pm 0.007$ \\
$0.029 \pm 0.028$ \\
$67.073 \pm 33.092$ \\
$158.753 \pm 13.614^{f}$ \\
$0.219 \pm 0.023$ \\
$28.813 \pm 1.585$ \\
$8.770 \pm 0.659^{f}$ \\
$0.013 \pm 0.008$ \\
$0.054 \pm 0.006^{f}$ \\
$0.033 \pm 0.021$ \\
$6.689 \pm 1.106^{f}$ \\
$0.047 \pm 0.011$ \\
$0.046 \pm 0.011$ \\
$158.673 \pm 13.619^{f}$ \\
$0.519 \pm 0.251$ \\
$99.833 \pm 21.772^{\mathrm{e}}$ \\
\hline
\end{tabular}

Data are presented as meantS.E.M. $\left(n=5 ;{ }^{\text {a }} P \leq 0.05 ;{ }^{\text {b }} P \leq 0.01 ;{ }^{c} P \leq 0.001\right.$ for normal adipogenesis in comparison to day 0 and ${ }^{\mathrm{d}} P \leq 0.05$; ${ }^{\mathrm{e}} P \leq 0.01$; ${ }^{\mathrm{f}} P \leq$ 0.001 for comparing unexposed and androstenedione (AD)-exposed cells at day 8 ).

showing an alteration during normal adipogenesis or after androstenedione application (Fig. 5E). The progesterone receptor was detectable by sequencing, but the expression was too low for quantification by qRT.

\section{Discussion}

Adipose tissue has been recognized as an steroidogenic organ producing and transforming a wide range of steroid hormones in a complex network of steroidogenic enzymes $(22,23,24)$. The ability of de novo synthesis of steroid hormones from cholesterol depends on the presence of the mitochondrial cholesterol transport machinery and CYP11A1 that converts cholesterol into pregnenolone. In our study, StAR - one major component of the mitochondrial cholesterol transport machinery - and CYP11A1 only were present with increasing expression of CYP11A1 during SGBS adipogenesis. Simultaneously, cholesterol level in supernatants of maturating adipocytes decreased, and several steroid hormones were detectable, namely progesterone, androgens, estradiol and corticosteroids. Estrogen as well as the CYP19 expression was not altered during adipogenesis. Data from SGBS over an 8-21-day differentiation period showed a steady decrease of CYP19 (31), and the comparison of preadipocyte and adult adipocyte from primary human culture revealed a significant downregulation (32). Furthermore, we found estrogen receptors to be expressed in SGBS cells. Preadipocytes had only low copies of $E R \alpha, E R \beta$ and GPER with a significant increase during differentiation. The occurrence of estrogen receptors and their up-regulation during adipogenesis has already been described in SGBS and 3T3-L1 cells, assuming an involvement in the differentiation of preadipocytes $(33,34)$. An elevation of the androgen level was found solely at day 4 , whereas for the androgen interconverting HSD17B5 until day 8. This is in agreement with data of differentiating stromal preadipocytes from s.c. fat biopsies to mature adipocytes (35). As expected, we found the $A R$ to be expressed in maturing SGBS cells with a significant increase at all analysed time points. AR has already been demonstrated to be present in human adipocytes and SGBS cells $(33,36,37)$. An upregulated transcription but down-regulated activity of AR as a result of GR-activation is an essential step for inducing adipocyte differentiation driven by dexamethasone, an important supplement in the culture media used during adipogenesis $(25,26,38$, 39). Interestingly, GR expression did not change, which may have resulted from an already reached maximum induction by the culture media additive dexamethasone. At glucocorticoid level, corticosterone and both cortisol and cortisone, increased during overall normal maturation. Despite the fact of cortisol-supplementation in the culture media during adipogenesis $(25,26)$, SGBS cells secreted cortisol by themselves that displayed an up to 7-fold (day 4) and 6-fold (day 8) rise when compared to values at day 0 . HSD11B1, a bidirectional cortisolcortisone-interconverting enzyme (40), was upregulated only during induction stage. During 3T3-L1 adipogenesis HSD11B1 increased and an activation of expression was paralleled by human adipocyte differentiation $(27,40)$.

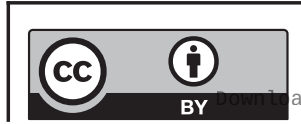

This work is licensed under a Creative Commons Attribution 4.0 International License. ded from Bioscientifica.com at 04/26/2023 01:00:50PM 
A
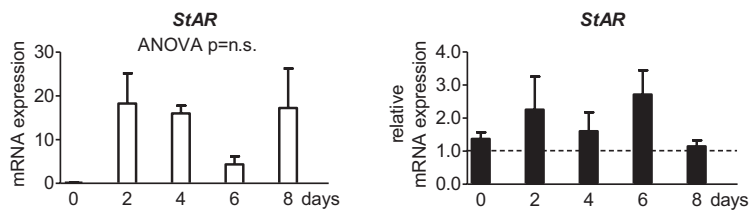

B
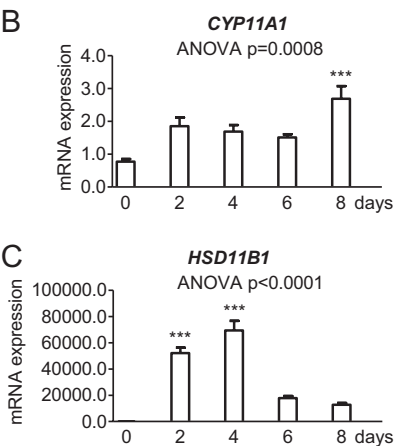

D

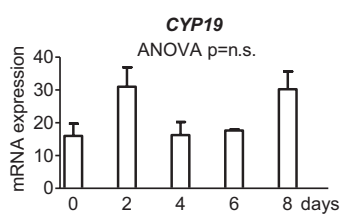

$\mathrm{E}$

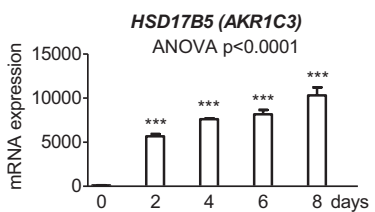

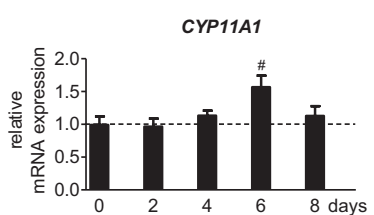

HSD11B1

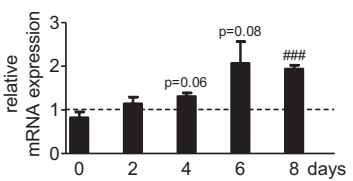

CYP19

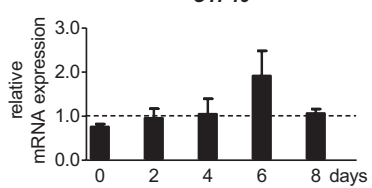

HSD17B5 (AKR1C3)

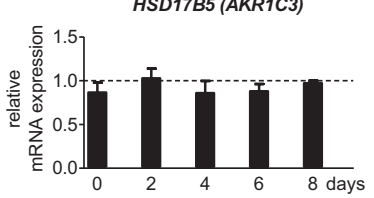

Figure 4

Gene expression of enzymes of the steroid hormone metabolism in the SGBS cell model during adipogenesis. The absolute mRNA expression of (A) the steroidogenic acute regulatory protein (StAR), (B) the cholesterol side-chain cleavage enzyme P450scc (CYP11A1), (C) 11 beta-hydroxysteroid dehydrogenase 1 (HSD11B1), (D) the cytochrome P450 aromatase (CYP19) and (E) the aldo-keto reductase family 1 member C3 AKR1C3 (HSD17B5) normalized to $10^{3}$ molecules of TBP as housekeeping gene was evaluated in SGBS cells during adipogenic differentiation under normal conditions (left). Additionally, AD stimulation is described in relation to the corresponding AD-free control level indicated as dotted line (right) ( $n=4$; * $P \leq 0.05$; ** $P \leq 0.01$; *** $P \leq 0.001$ for normal adipogenesis in comparison to day 0 and $\# P \leq 0.05$; \#\# $P \leq 0.01$; \#\# $P \leq 0.001$ for comparing unexposed and AD-exposed cells).

On the base of these results we suggest, that SGBS adipocytes have a functional de novo steroid hormone biosynthesis and are a suitable steroidogenic fat cell model to simulate diverse pathological scenarios.

Investigating the hypothesis of a substantial contribution to androgen synthesis by adipose tissue (10), we treated maturing SGBS preadipocytes with an exceeding concentration of androstenedione, the precursor of active steroid hormones. First, androstenedione did not affect proliferation of cells or the secretion of leptin and adiponectin. Both adipokines were taken as validation markers for a correct maturation of the SGBS
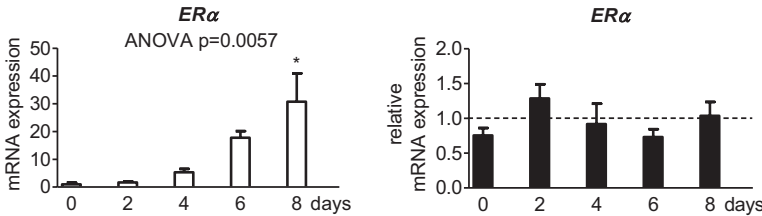

B
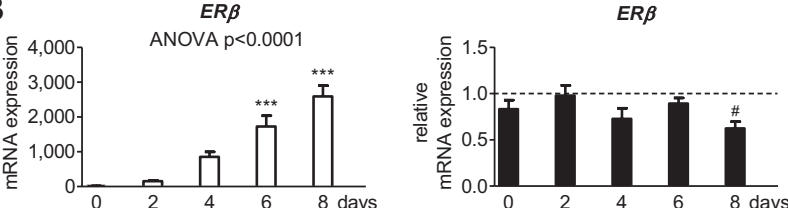

C
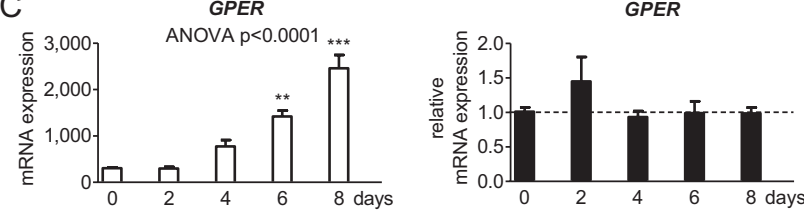

D
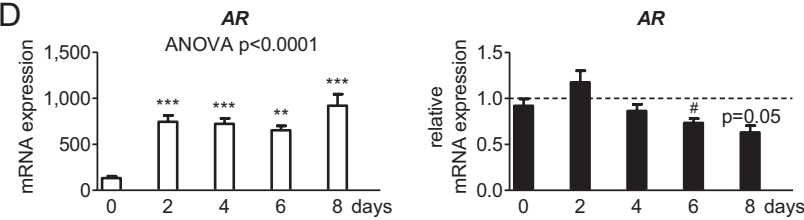

$\mathrm{E}$
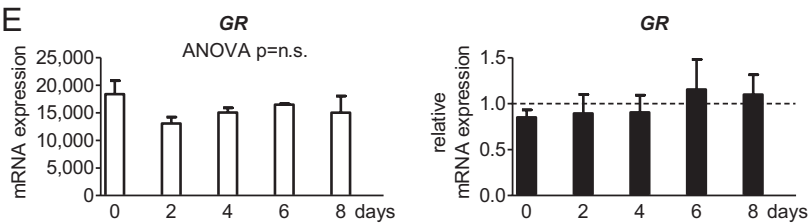

Figure 5

Influence of androstenedione on estrogen receptors in the SGBS cell model. The absolute mRNA expression of (A) estrogen receptor alpha $(E R \alpha)$ and $(B)$ beta $(E R \beta)$ as well as (C) the $G$ protein-coupled estrogen receptor 1 (GPER), (D) the androgen receptor $(A R)$ and $(E)$ the glucocorticoid receptor (GR) normalized to $10^{3}$ molecules of TBP as housekeeping gene was evaluated in SGBS cells during adipogenic differentiation under normal conditions (left). Additionally, AD stimulation is described in relation to the corresponding AD-free control level indicated as dotted line (right) $(n=4 ; * P \leq 0.05 ; * * P \leq 0.01$; *** $P \leq 0.001$ for normal adipogenesis in comparison to day 0 and $\# P \leq 0.05$; \#\# $P \leq$ 0.01; \#\# $P \leq 0.001$ for comparing unexposed and AD-exposed cells).

preadipocytes as previously described (26). Studies on the SGBS model and primary human s.c. adipocytes with active androgens demonstrated no significant influence on proliferation, but a negative impact on differentiation through an AR-driven pathway reducing leptin but not adiponectin secretion $(33,37,41,42,43)$. Interestingly, the precursor dehydroepiandrosterone impaired cell proliferation without significant effect on differentiation (44). Analyses of the insulin-regulated glucose transporter

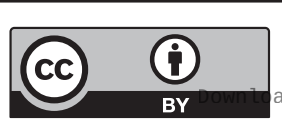

This work is licensed under a Creative Commons Attribution 4.0 International License. 
GLUT4 revealed an upregulated gene expression (data not shown) and a predominant membrane-bound distribution under normal conditions, reasoned by the association of adipogenesis with increasing insulinsensitivity $(45,46)$. However, androstenedione had no impact on GLUT4. Treatment of the human s.c. cell line Chub7 with dehydroepiandrosterone caused an elevation of the basal, but not insulin-stimulated glucose-uptake (47). Quantification of the lipid accumulation in SGBS revealed a $40 \%$ elevated triglyceride content within the adult adipocytes under androstenedione treatment. In this regard, we found no influence of androstenedione on PLIN1 and HSL (components of the lipid droplets). In contrast to our results, potent androgens decreased lipid content in the same cell model and in primary human adipocytes $(37,43)$. Another study demonstrated no alteration of the basal but reduced cAMP-stimulated lipolysis with no effect on PLIN1 (48). PLIN1 is a highly expressed adipocyte-specific protein on the surface of lipid vesicles associated with the stored triglyceride content. Thereby, it contributes to homeostasis in lipid metabolism by blocking basal and mediating hormonestimulated lipolysis $(49,50,51,52)$.

Second, as the cholesterol level did not change, we assume the employed androstenedione did not activate the de novo steroid hormone synthesis, but rather was converted into othersteroids. The secretion of progesterone, testosterone (additionally dihydrotestosterone as well as the less active androgenic metabolites androsterone and etiocholanolone) and cortisone was accelerated by androstenedione. A new finding is the androstenedionemediated induction of the progesterone level as well as of the CYP11A1 expression in adipocytes. Interestingly, granulosa cells synthesize significantly higher levels of estrone and estradiol without changes of progesterone and androgen under low concentrations of androgen precursors with the limitation that higher concentrations would lead to increasing androgen levels by possibly masking the precursor effect (53). However, the treatment of granulosa cells with a potent androgen accelerates progesterone synthesis and enhances the expression of StAR and CYP11A1, but otherwise had no significant effect on estrogen synthesis (54). Downstream of progesterone to glucocorticoids we found alterations as followed: 11-deoxycortisol and furthermore, cortisone - but not cortisol - were elevated at day 8. In this regard, HSD11B1 was changed in the same way. HSD11B1-expression was found to be increased in in vitro androgen-treated male preadipocytes (32). Furthermore, HSD11B1 expression was positively correlated in s.c. fat of obese women with circulating testosterone (55). As HSD11B1 is responsible for the bidirectional cortisol-cortisone-interconversion, in adipose tissue the dehydrogenase activity (cortisol to cortisone) predominates the oxidoreductase activity (cortisone to cortisol). Accordingly, this predominance stood against previous assumption of a linear relation between the intra-adipose glucocorticoid and HSD11B1 level (56). In Chub-S7 preadipocytes, the HSD11B1 expression was reduced by dehydroepiandrosterone. Simultaneously, its enzyme activity revealed a decreased oxidoreductase activity with concurrent increased dehydrogenase activity (47). The switch to the predominant conversion to hormonally inactive glucocorticoids implicates a metabolic protective mechanism (57).

Summing up, the present study describes a functional de novo steroid hormone biosynthesis for the SGBS adipogenic cell model. Thereby, our results confirm the steroidogenic activity of adipocytes and the function of adipose tissue as a steroidogenic organ. Treatment with the androgen precursor androstenedione had no influence on the adipogenic differentiation to mature SGBS adipocytes and did not cause lipolytic effects. However, androstenedione application significantly altered steroidogenesis: to our knowledge, we are the first to show an induction of progesterone production in adipocytes. Besides this, downstream steroid hormone secretion points towards an upregulated androgen and glucocorticoid pathway, whereas the estrogenic pathway seemed not to be affected. Our current data suggest that adipocytes do not initiate androgen excess, but contribute to an already persisting androgen excess.

Despite a multitude of advantages, in vitro cell models like SGBS are also subject to limitations. One obvious limitation is the lack of systemic crosstalk, like hormonal interactions, which occur in a whole organism. Whether differentiation and proliferation processes in cell models mimic the precise course in vivo seems difficult to assess. Furthermore, the obtained results in single cell cultures cannot directly be transferred to in vivo situation. Nevertheless, the SGBS model is clearly closer to other human systems than murine models. The SGBS model allows identifying the main players and central signaling pathways with their regulations.

\section{Declaration of interest}

The authors declare that there is no conflict of interest that could be perceived as prejudicing the impartiality of the research reported.

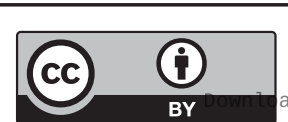

This work is licensed under a Creative Commons Attribution 4.0 International License. ded from Bioscientifica.com at 04/26/2023 01:00:50PM 


\section{Funding}

Jana Ernst and Kristina Schädlich were supported by the Roux Programme of the Faculty of Medicine, Martin Luther University Halle-Wittenberg (JE FKZ 29/11, KS FKZ 26/06). We acknowledge the financial support within the funding programme Open Access Publishing by the German Research Foundation (DFG).

\section{Author contribution statement}

J E: acquisition, analysis and interpretation of data, conception and design of the study, drafting the article and final approval. K G: acquisition, analysis and interpretation of data, final approval. F B K: acquisition, analysis and interpretation of data, revising the article and final approval. U E R-K: analysis of data, final approval. M W: contribution to the technique of SGBS cell culture, discussion of the data, final approval. F D: project leader, revising the article and final approval. K S: project leader, conception and design of the study, revising the article and final approval.

Acknowledgements

The authors thank Christine Froehlich for excellent technical assistance.

\section{References}

1 Organisation for Economic Co-operation and Development. OECD obesity update 2017. Paris, France: OECD, 2017. (https://www.oecd. org/health/obesity-update.htm)

2 World Health Organization. Controlling the global obesity epidemic. Geneva, Switzerland: WHO, 2003. (https://www.who.int/nutrition/ topics/obesity/en/)

3 Armitage JA, Poston L \& Taylor PD. Developmental origins of obesity and the metabolic syndrome: the role of maternal obesity. Frontiers of Hormone Research 200836 73-84. (https://doi. org/10.1159/00011535)

4 Fowden AL, Giussani DA \& Forhead AJ. Intrauterine programming of physiological systems: causes and consequences. Physiology 200621 29-37. (https://doi.org/10.1152/physiol.00050.2005)

5 Gluckman PD, Hanson MA, Cooper C \& Thornburg KL. Effect of in utero and early-life conditions on adult health and disease. New England Journal of Medicine 2008359 61-73. (doi:10.1056/ NEJMra0708473

6 Dunaif A. Insulin resistance and the polycystic ovary syndrome: mechanism and implications for pathogenesis. Endocrine Reviews 199718 774-800. (https://doi.org/10.1210/edrv.18.6.0318)

7 Anderson AD, Solorzano CMB \& McCartney CR. Childhood obesity and its impact on the development of adolescent PCOS. Seminars in Reproductive Medicine 201432 202-213. (https://doi. org/10.1055/s-0034-1371092)

8 Payne AH \& Hales DB. Overview of steroidogenic enzymes in the pathway from cholesterol to active steroid hormones. Endocrine Reviews 200425 947-970. (https://doi.org/10.1210/er.2003-0030)

9 Fehér T \& Bodrogi L. A comparative study of steroid concentrations in human adipose tissue and the peripheral circulation. Clinica Chimica Acta 1982126 135-141. (https://doi.org/10.1016/00098981(82)90029-8)

10 Wagner IV, Sahlin L, Savchuk I, Klöting N, Svechnikov K \& Söder O. Adipose tissue is a potential source of hyperandrogenism in obese female rats. Obesity 201826 1161-1167. (https://doi.org/10.1002/ oby.22198)

11 Yamamoto A, Kakutani N, Yamamoto K, Kamiura T \& Miyakoda H. Steroid hormone profiles of urban and tidal rivers using LC/MS/ MS equipped with electrospray ionization and atmospheric pressure photoionization sources. Environmental Science and Technology 2006 40 4132-4137. (https://doi.org/10.1021/es052593p)

12 Vulliet E \& Cren-Olivé C. Screening of pharmaceuticals and hormones at the regional scale, in surface and groundwaters intended to human consumption. Environmental Pollution 2011159 2929-2934. (https://doi.org/10.1016/j.envpol.2011.04.033)

13 Stavreva DA, George AA, Klausmeyer P, Varticovski L, Sack D, Voss TC, Schiltz RL, Blazer VS, Iwanowicz LR \& Hager GL. Prevalent glucocorticoid and androgen activity in US water sources. Science Reports 20122 937. (https://doi.org/10.1038/srep00937)

14 Nohara K, Waraich RS, Liu S, Ferron M, Waget A, Meyers MS Karsenty G, Burcelin R \& Mauvais-Jarvis F. Developmental androgen excess programs sympathetic tone and adipose tissue dysfunction and predisposes to a cardiometabolic syndrome in female mice. American Journal of Physiology: Endocrinology and Metabolism $2013 \mathbf{3 0 4}$ E1321-E1330. (https://doi.org/10.1152/ajpendo.00620.2012)

15 Nohara K, Liu S, Meyers MS, Waget A, Ferron M, Karsenty G, Burcelin R \& Mauvais-Jarvis F. Developmental androgen excess disrupts reproduction and energy homeostasis in adult male mice. Journal of Endocrinology 2013219 259-268. (https://doi.org/10.1530/JOE-13-0230)

16 Mauvais-Jarvis F. Developmental androgenization programs metabolic dysfunction in adult mice: clinical implications. Adipocyte 20143 151-154. (https://doi.org/10.4161/adip.27746)

17 Eisner JR, Dumesic DA, Kemnitz JW, Colman RJ \& Abbott DH. Increased adiposity in female rhesus monkeys exposed to androgen excess during early gestation. Obesity Research 200311 279-286. (https://doi.org/10.1038/oby.2003.42)

18 Bruns CM, Baum ST, Colman RJ, Eisner JR, Kemnitz JW, Weindruch R \& Abbott DH. Insulin resistance and impaired insulin secretion in prenatally androgenized male rhesus monkeys. Journal of Clinical Endocrinology and Metabolism 200489 6218-6223. (https://doi. org/10.1210/jc.2004-0918)

19 Bruns CM, Baum ST, Colman RJ, Dumesic DA, Eisner JR, Jensen MD, Whigham LD \& Abbott DH. Prenatal androgen excess negatively impacts body fat distribution in a nonhuman primate model of polycystic ovary syndrome. International Journal of Obesity $2007 \mathbf{3 1}$ 1579-1585. (https://doi.org/10.1038/sj.ijo.0803638)

20 Sir-Petermann T, Codner E, Pérez V, Echiburú B, Maliqueo M, Ladrón de Guevara A, Preisler J, Crisosto N, Sánchez F, Cassorla F, et al. Metabolic and reproductive features before and during puberty in daughters of women with polycystic ovary syndrome. Journal of Clinical Endocrinology and Metabolism 200994 1923-1930. (https:// doi.org/10.1210/jc.2008-2836)

21 Recabarren SE, Rojas-García PP, Recabarren MP, Alfaro VH, Smith R, Padmanabhan, V \& Sir-Petermann T. Prenatal testosterone excess reduces sperm count and motility. Endocrinology 2008149 6444-6448. (https://doi.org/10.1210/en.2008-0785)

22 Tchernof A, Mansour MF, Pelletier M, Boulet M-M, Nadeau M, van Luu-The. Updated survey of the steroid-converting enzymes in human adipose tissues. Journal of Steroid Biochemistry and Molecular Biology 2015147 56-69. (https://doi.org/10.1016/j.jsbmb.2014.11.011)

23 O'Reilly MW, House PJ \& Tomlinson JW. Understanding androgen action in adipose tissue. Journal of Steroid Biochemistry and Molecular Biology 2014143 277-284. (https://doi.org/10.1016/j. jsbmb.2014.04.008)

24 Li J, Papadopoulos V \& Vihma V. Steroid biosynthesis in adipose tissue. Steroids 2015103 89-104. (https://doi.org/10.1016/j. steroids.2015.03.016)

25 Wabitsch M, Brenner RE, Melzner I, Braun M, Möller P, Heinze E, Debatin KM \& Hauner H. Characterization of a human preadipocyte cell strain with high capacity for adipose differentiation. International Journal of Obesity and Related Metabolic Disorders 200125 8-15.

26 Fischer-Posovszky P, Newell FS, Wabitsch M, Tornqvist HE. Human SGBS cells-a unique tool for studies of human fat cell biology. Obesity Facts 20081 184-189. (https://doi.org/10.1159/000145784)

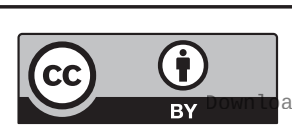

This work is licensed under a Creative Commons Attribution 4.0 International License. ded from Bioscientifica,com at 04/26/2023 01:00:50PM 
27 Li J, Daly E, Campioli E, Wabitsch M \& Papadopoulos V. De novo synthesis of steroids and oxysterols in adipocytes. Journal of Biological Chemistry 2014289 747-764. (https://doi.org/10.1074/jbc. M113.534172)

28 Bodles AM, Banga A, Rasouli N, Ono F, Kern PA \& Owens RJ. Pioglitazone increases secretion of high-molecular-weight adiponectin from adipocytes. American Journal of Physiology: Endocrinology and Metabolism 2006291 E1100-E1105. (https://doi. org/10.1152/ajpendo.00187.2006)

29 Grigem S, Fischer-Posovszky P, Debatin KM, Loizon E, Vidal H \& Wabitsch M. The effect of the HIV protease inhibitor ritonavir on proliferation, differentiation, lipogenesis, gene expression and apoptosis of human preadipocytes and adipocytes. Hormone and Metabolic Research 200537 602-609. (https://doi. org/10.1055/s-2005-870526)

30 Körner A, Wabitsch M, Seidel B, Fischer-Posovszky P, Berthold A, Stumvoll M, Blüher M, Kratzsch J \& Kiess W. Adiponectin expression in humans is dependent on differentiation of adipocytes and downregulated by humoral serum components of high molecular weight. Biochemical and Biophysical Research Communications 2005337 540-550. (https://doi.org/10.1016/j.bbrc.2005.09.064)

31 McInnes KJ, Brown KA, Knower KC, Chand AL, Clyne CD \& Simpson ER. Characterisation of aromatase expression in the human adipocyte cell line SGBS. Breast Cancer Research and Treatment 2008 112 429-435. (https://doi.org/10.1007/s10549-007-9883-2)

32 Dieudonné M-N, Sammari A, Dos Santos E, Leneveu M-C, Giudicelli Y \& Pecquery R. Sex steroids and leptin regulate 11 betahydroxysteroid dehydrogenase I and P450 aromatase expressions in human preadipocytes: sex specificities. Journal of Steroid Biochemistry and Molecular Biology 200699 189-196. (https://doi.org/10.1016/j. jsbmb.2006.01.007)

33 Horenburg S, Fischer-Posovszky P, Debatin K-M \& Wabitsch M. Influence of sex hormones on adiponectin expression in human adipocytes. Hormone and Metabolic Research 200840 779-786. (https://doi.org/10.1055/s-0028-1083780)

34 Zhu P, Yuen JML, Sham KWY \& Cheng CHK. GPER mediates the inhibitory actions of estrogen on adipogenesis in 3T3-L1 cells through perturbation of mitotic clonal expansion. General and Comparative Endocrinology 2013193 19-26. (https://doi. org/10.1016/j.ygcen.2013.07.004)

35 Quinkler M, Sinha B, Tomlinson JW, Bujalska IJ, Stewart PM \& Arlt W. Androgen generation in adipose tissue in women with simple obesity-a site-specific role for 17beta-hydroxysteroid dehydrogenase type 5. Journal of Endocrinology 2004183 331-342. (https://doi. org/10.1677/joe.1.05762)

36 Dieudonne MN, Pecquery R, Boumediene A, Leneveu MC \& Giudicelli Y. Androgen receptors in human preadipocytes and adipocytes: regional specificities and regulation by sex steroids. American Journal of Physiology 1998274 C1645-C1652. (https://doi. org/10.1152/ajpcell.1998.274.6.C1645)

37 Gupta V, Bhasin S, Guo W, Singh R, Miki R, Chauhan P, Choong K, Tchkonia T, Lebrasseur NK, Flanagan JN, et al. Effects of dihydrotestosterone on differentiation and proliferation of human mesenchymal stem cells and preadipocytes. Molecular and Cellular Endocrinology 2008296 32-40. (https://doi.org/10.1016/j. mce.2008.08.019)

38 Chapman AB, Knight DM \& Ringold GM. Glucocorticoid regulation of adipocyte differentiation: hormonal triggering of the developmental program and induction of a differentiationdependent gene. Journal of Cell Biology 1985101 1227-1235. (https:// doi.org/10.1083/jcb.101.4.1227)

39 Hartig SM, He B, Newberg JY, Ochsner SA, Loose DS, Lanz RB, McKenna NJ, Buehrer BM, McGuire SE, Marcelli M, et al. Feedforward inhibition of androgen receptor activity by glucocorticoid action in human adipocytes. Chemical Biology 201219 1126-1141. (https://doi.org/10.1016/j.chembiol.2012.07.020)
40 Bujalska IJ, Walker EA, Tomlinson JW, Hewison M \& Stewart PM. 11Beta-hydroxysteroid dehydrogenase type 1 in differentiating omental human preadipocytes: from de-activation to generation of cortisol. Endocrine Research 200228 449-461.

41 Anderson LA, McTernan PG, Barnett AH \& Kumar S. The effects of androgens and estrogens on preadipocyte proliferation in human adipose tissue: influence of gender and site. Journal of Clinical Endocrinology and Metabolism 200186 5045-5051. (https://doi. org/10.1210/jcem.86.10.7955)

42 Chazenbalk G, Singh P, Irge D, Shah A, Abbott DH \& Dumesic DA Androgens inhibit adipogenesis during human adipose stem cell commitment to preadipocyte formation. Steroids $2013 \mathbf{7 8} 920-926$. (https://doi.org/10.1016/j.steroids.2013.05.001)

43 Kraus M, Greither T, Wenzel C, Bräuer-Hartmann D, Wabitsch M \& Behre HM. Inhibition of adipogenic differentiation of human SGBS preadipocytes by androgen-regulated microRNA miR-375. Molecular and Cellular Endocrinology 2015414 177-185. (https://doi. org/10.1016/j.mce.2015.07.026)

44 Rice SPL, Zhang L, Grennan-Jones F, Agarwal N, Lewis MD, Rees DA \& Ludgate M. Dehydroepiandrosterone (DHEA) treatment in vitro inhibits adipogenesis in human omental but not subcutaneous adipose tissue. Molecular and Cellular Endocrinology 2010320 51-57. (https://doi.org/10.1016/j.mce.2010.02.017)

45 Hauner H, Röhrig K, Spelleken M, Liu LS \& Eckel J. Development of insulin-responsive glucose uptake and GLUT4 expression in differentiating human adipocyte precursor cells. International Journal of Obesity and Related Metabolic Disorders 199822 448-453.

46 Pederson T \& Rondinone CM. Regulation of proteins involved in insulin signaling pathways in differentiating human adipocytes. Biochemical and Biophysical Research Communications 2000276 162-168. (https://doi.org/10.1006/bbrc.2000.3429)

47 McNelis JC, Manolopoulos KN, Gathercole LL, Bujalska IJ, Stewart PM, Tomlinson JW \& Arlt W. Dehydroepiandrosterone exerts antiglucocorticoid action on human preadipocyte proliferation, differentiation, and glucose uptake. American Journal of Physiology: Endocrinology and Metabolism 2013305 E1134-E1144. (https://doi org/10.1152/ajpendo.00314.2012)

48 Dicker A, Rydén M, Näslund E, Muehlen IE, Wirén M, Lafontan M \& Arner P. Effect of testosterone on lipolysis in human pre-adipocytes from different fat depots. Diabetologia 200447 420-428. (https://doi. org/10.1007/s00125-003-1324-0)

49 Sun Z, Gong J, Wu H, Xu W, Wu L, Xu D, Gao J, Wu J-W, Yang H, Yang M, et al. Perilipin1 promotes unilocular lipid droplet formation through the activation of Fsp27 in adipocytes. Nature Communications 20134 1594. (https://doi.org/10.1038/ncomms2581)

50 Yang X, Heckmann BL, Zhang X, Smas CM \& Liu J. Distinct mechanisms regulate ATGL-mediated adipocyte lipolysis by lipid droplet coat proteins. Molecular Endocrinology 201327 116-126. (https://doi.org/10.1210/me.2012-1178)

51 Zhang S, Liu G, Xu C, Liu L, Zhang Q, Xu Q, Jia H, Li X \& Li X. Perilipin 1 mediates lipid metabolism homeostasis and inhibits inflammatory cytokine synthesis in bovine adipocytes. Frontiers in Immunology 20189 467. (https://doi.org/10.3389/fimmu.2018.00467)

52 Natori Y, Nasui M \& Kihara-Negishi F. Neu1 sialidase interacts with perilipin 1 on lipid droplets and inhibits lipolysis in 3T3-L1 adipocytes. Genes to Cells 201722 485-492. (https://doi.org/10.1111/ gtc.12490)

53 ElBeltagy K, Honda K-i, Ozaki K, Misugi T, Tokuyama O, Kimura M, Kira Y \& Ishiko O. In vitro effect of dehydroepiandrosterone sulfate on steroid receptors, aromatase, cyclooxygenase-2 expression, and steroid hormone production in preovulatory human granulosa cells. Fertility and Sterility 200788 (Supplement) 1135-1142. (https://doi. org/10.1016/j.fertnstert.2007.01.008)

54 Hasegawa T, Kamada Y, Hosoya T, Fujita S, Nishiyama Y, Iwata N, Hiramatsu Y \& Otsuka F. A regulatory role of androgen in ovarian steroidogenesis by rat granulosa cells. Journal of Steroid Biochemistry

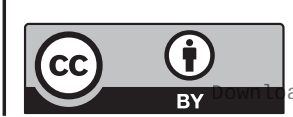

This work is licensed under a Creative Commons Attribution 4.0 International License. ded from Bioscientifica com at 04/26/2023 01:00:50PN 
and Molecular Biology 2017172 160-165. (https://doi.org/10.1016/j. jsbmb.2017.07.002)

55 Gambineri A, Fanelli F, Tomassoni F, Munarini A, Pagotto U, Andrew R, Walker BR \& Pasquali R. Tissue-specific dysregulation of $11 \beta$-hydroxysteroid dehydrogenase type 1 in overweight/obese women with polycystic ovary syndrome compared with weightmatched controls. European Journal of Endocrinology 2014171 47-57. (https://doi.org/10.1530/EJE-13-1030)
56 Hughes KA, Manolopoulos KN, Iqbal J, Cruden NL, Stimson RH, Reynolds RM, Newby DE, Andrew R, Karpe F \& Walker BR. Recycling between cortisol and cortisone in human splanchnic, subcutaneous adipose, and skeletal muscle tissues in vivo. Diabetes $2012 \mathbf{6 1}$ 1357-1364. (https://doi.org/10.2337/db11-1345)

57 Kershaw EE \& Flier JS. Adipose tissue as an endocrine organ. Journal of Clinical Endocrinology and Metabolism 200489 2548-2556. (https:// doi.org/10.1210/jc.2004-0395)

Received in final form 28 May 2020

Accepted 3 June 2020

Accepted Manuscript published online 4 June 2020

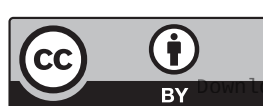

This work is licensed under a Creative Commons Attribution 4.0 International License.

ded from Bioscientifica.com at 04/26/2023 01:00:50PM via free access 